\title{
N-Acetylcysteine for the Prevention of Contrast-induced Nephropathy
}

\section{A Systematic Review and Meta-analysis}

\author{
Raymond Liu, MD, ${ }^{1,2}$ Deepu Nair, MD, ${ }^{1,2}$ Joachim Ix, MD, ${ }^{1,3}$ Dan H. Moore PhD, ${ }^{4,5}$ \\ Stephen Bent, MD $1,3,6$ \\ 'Department of Medicine, University of California, San Francisco, CA, USA; ${ }^{2}$ Veterans Affairs PRIME Program and ${ }^{3}$ Department of \\ Medicine, San Francisco Veterans Affairs Medical Center, San Francisco, CA, USA; ${ }^{4}$ Research Institute, California Pacific Medical Center, \\ San Francisco, CA, USA; ${ }^{5}$ Department of Epidemiology and Biostatistics and ${ }^{6}$ Osher Center for Integrative Medicine, University of \\ California, San Francisco, CA, USA
}

\begin{abstract}
OBJECTIVE: Contrast-induced nephropathy is a common cause of acute renal failure in hospitalized patients. Although patients are often given $\mathrm{N}$-acetylcysteine to prevent renal injury from contrast agents, there are no clear guidelines supporting its use. We conducted a systematic review to determine whether administering $\mathrm{N}$-acetylcysteine around the time of contrast administration reduces the risk of contrastinduced nephropathy.
\end{abstract}

DESIGN: We searched MEDLINE, EMBASE, the Cochrane Collaboration Database, bibliographies of retrieved articles, and abstracts of conference proceedings, and consulted with experts to identify relevant studies. Randomized controlled trials of $\mathrm{N}$-acetylcysteine in hospitalized patients receiving contrast were included. Studies were excluded if they did not report change in creatinine or incidence of contrast-induced nephropathy at 48 hours.

MEASUREMENTS AND MAIN RESULTS: Nine randomized controlled trials satisfied all inclusion criteria and were included in the analysis. The difference in mean change in creatinine between the N-acetylcysteine-treated group and controls was $-0.27 \mathrm{mg} / \mathrm{dl}$ (95\% confidence interval $[\mathrm{CI}],-0.43$ to -0.11$)$. The relative risk of developing contrastinduced nephropathy was 0.43 (95\% CI, 0.24 to 0.75$)$ in subjects randomized to $\mathrm{N}$-acetylcysteine. Significant heterogeneity existed among studies, suggesting differences in patient populations or study methodology not identified by sensitivity analyses. The incidence of dialysis was rare $(0.2 \%)$

CONCLUSIONS: Our findings suggest that $\mathrm{N}$-acetylcysteine helps prevent declining renal function and contrast-induced nephropathy. While $\mathrm{N}$-acetylcysteine is inexpensive and nontoxic, undeviating insistence for dosing at least 12 hours in advance of contrast exposure may delay diagnostic and therapeutic procedures. Future studies are needed to address the longer-term clinical outcomes and cost-effectiveness of this agent.

KEY WORDS: N-acetylcysteine; contrast-induced nephropathy; acute renal failure; kidney disease.

DOI: $10.1111 /$ j. 1525-1497.2005.30323.x

J GEN INTERN MED 2005; 20:193-200.

$\mathrm{T}$ here are over 10 million intravascular contrast procedures performed each year, with more than 1 million patients undergoing coronary angiography annually., Contrast-induced nephropathy (CIN) is a common complication of these procedures, with an incidence ranging from zero to over $50 \%$, depending on the population studied. ${ }^{3,4}$

Patients who develop CIN have an increased risk of morbidity, mortality, and length of hospitalization. ${ }^{1,5-9}$ Several different risk factors for CIN have been identified, including

Accepted for publication July 1, 2004

Address correspondence and requests for reprints to Dr. Liu: San Francisco VA Medical Center, 111-A1, 4150 Clement Street, San Francisco, CA 94121 (e-mail: rliu3@yahoo.com). chronic renal insufficiency, ${ }^{10,11}$ diabetes, ${ }^{1,3,4,12}$ and high-contrast dose. ${ }^{1,4,11,13-18}$

Though the exact mechanism by which contrast administration causes CIN is not known, many potential mechanisms have been proposed. Direct tubular toxicity with generation of oxygen free radicals as well as renal vasoconstriction via a nitric oxide or endothelin pathway have been proposed. ${ }^{19,20}$ The free radical mechanism of direct tubular toxicity has led several investigators to focus on the use of $\mathrm{N}$-acetylcysteine (NAC) for the prevention of CIN, as NAC has been previously shown to have antioxidant properties. ${ }^{21}$

Some clinical trials have demonstrated a protective effect of NAC in the prevention of CIN,${ }^{22-29}$ while others have not. ${ }^{2,30-35}$ There are currently no established national guidelines on whether patients should be given NAC and use of this agent varies widely. ${ }^{36}$ We therefore performed a meta-analysis of published human randomized controlled trials in order to determine whether the administration of NAC around the time of contrast delivery is protective against CIN, and to better quantify the magnitude of this effect.

\section{METHODS}

\section{Literature Review}

We performed a literature search of MEDLINE (January 1966August 2003), EMBASE (1974-August 2003), and the Cochrane Collaboration Database (1996-August 2003) using the keywords acetylcysteine, kidney disease, and clinical trials. In addition, a manual search of abstracts of conference proceedings since 2000 from the National Kidney Foundation, the American Society of Nephrology, the American Heart Association, and the American College of Cardiology was performed. This strategy was combined with a manual search of reference lists from identified articles and consultation with experts.

Our analysis was limited to randomized controlled trials examining the efficacy of NAC for the prevention CIN. In order to be included, studies were required to report sufficient data to calculate either a mean change in creatinine in each group after contrast administration or the percentage of patients developing CIN.

\section{Data Extraction}

Two of the authors (D.N. and J.I.) independently reviewed each of the identified studies to determine eligibility and perform data abstraction. Using a standardized form, the reviewers recorded sample size, study setting, patient characteristics, methods and timing of NAC administration, presence of and type of prehydration, baseline and final creatinine, and definition of CIN and all other reported outcome variables. All 
studies were assessed for quality by evaluating the appropriateness of randomization, allocation concealment, and blinding technique (patients and providers). High-quality studies were defined as complying with 2 or more of these quality features. If there was no information in the study regarding the quality criterion, the study was not credited with that criterion. A third author (R.L.) adjudicated any discrepancies noted between the two reviewers.

\section{Statistical Methods}

For the primary outcome measure-the difference in mean change in creatinine in patients given NAC versus patients given placebo-we performed a meta-analysis using a random effects model for continuous variables. For the secondary outcome measure, risk of developing CIN or requiring dialysis, we used a random effects model, weighting the summary measure from each study by the inverse variance for each study. Stata (Stata Statistical Software, Release 8.0, Stata Corporation, College Station, TX) was used to carry out the analyses. To test for publication bias, we used the Stata program Metabias, which performs the Begg and Mazumdar adjusted rank correlation test for publication bias and performs the Egger et al. regression asymmetry test for publication bias. ${ }^{37,38}$ The Begg adjusted rank correlation test is a direct statistical analog of the visual funnel graph. The Begg and Mazumdar procedure tests for publication bias by determining whether there is a significant correlation between the effect estimates and their variances. ${ }^{37}$ Metabias carries out this test by, first, standardizing the effect estimates to stabilize the variances and, second, performing an adjusted rank correlation test based on Kendall's $\tau$ (Stata Statistical Software, Release 8.0).

To test for heterogeneity, we calculated a $\chi^{2}$ statistic that is the sum of the squared differences between each study and the summary mean divided by the variance, that is, $\Sigma\left(\right.$ study $_{\mathrm{i}}-$ mean $^{2} /$ var $_{\mathrm{i}} \cdot{ }^{39}$ Assessment of the relative value of NAC in liquid or tablet form could not be made as the specific formulations were not generally reported in the studies.

\section{RESULTS}

\section{Study Selection}

The computerized database search identified 10 articles, and 7 more were identified from conference abstracts, review of bibliographies, and contact with experts (Table 1). Four studies were not randomized and were therefore excluded. ${ }^{2,24,25,31}$ Two studies had active treatment arms but no control groups and were therefore excluded. ${ }^{40,41}$ One randomized trial was excluded from analysis because it did not include data on outcomes. ${ }^{30}$ Another randomized trial was excluded because it reported data in subgroups only and did not provide sufficient information to calculate mean change in creatinine or the incidence of CIN. ${ }^{32}$ This left 9 randomized controlled trials of NAC, which met all eligibility criteria and were included in the meta-analysis (Table 1).

\section{Study Description}

The 9 studies selected took place from 2000 to 2003 and varied in size from 54 to 200 patients, with a total of 1,028 patients available for analysis. A total of 33 patients was lost to followup (3\%).
All studies were performed on hospitalized patients with an average age of 64 to 74 . With the exception of one study, ${ }^{28}$ patients received intra-arterial contrast, primarily for cardiac catheterization. Where reported, the populations studied had a high prevalence of comorbidities. The prevalence of significant illnesses varied as follows: diabetes $32 \%-64 \%$, congestive heart failure $0 \%-57 \%$, and hypertension $39 \%-92 \%$. The mean baseline creatinine varied from 1.35 to $2.8 \mathrm{mg} / \mathrm{dl}$. Medication use varied as follows: diuretics $20 \%-70 \%$ and ACE inhibitor $14 \%-65 \%$.

The method of NAC administration differed between studies. N-acetylcysteine was administered orally in all studies except one. ${ }^{29}$ No individual dose of oral NAC was less than $400 \mathrm{mg}$. The highest dose of oral NAC was $1,200 \mathrm{mg}$. Oral doses were given over varying amounts of time. The most common dose was $600 \mathrm{mg}$ NAC by mouth, and the most common schedule was twice a day, 3 doses before and 1 dose after the procedure (Table 1).

The use of prehydration was included in all studies, but also varied considerably. The most common dose was $0.45 \%$ normal saline at $1 \mathrm{ml} / \mathrm{kg} /$ hour. The most common schedule of hydration was for 12 hours before the procedure and 12 hours after the procedure. All studies that reported data used a low osmolar contrast agent, but the dose varied widely. The lowest average dose reported was $75 \mathrm{ml}$, and the highest dose was $238 \mathrm{ml}$. The total amount of saline given was not consistently reported.

There were varying definitions of CIN. Most studies used either a $25 \%$ increase in serum creatinine or a $0.5 \mathrm{mg} / \mathrm{dl}$ increase in serum creatinine. Placebos were used in 6 of the studies but varied widely and included matching tablets, orange juice, normal saline, and ginger ale. Study quality ranged from 0 to 2 out of a total possible score of 4 , with significant variation in the quality marker criteria that were satisfied (Table 1).

\section{Summary Effects}

Eight randomized studies reported the main outcome measure, mean change in creatinine at 48 hours. The summary estimate of these studies showed a difference in mean change in creatinine of $-0.27 \mathrm{mg} / \mathrm{dl}(95 \%$ confidence interval [CI], -0.43 to -0.11 ), as compared to placebo subjects, indicating a significant benefit in patients receiving NAC (Fig. 1). Heterogeneity was present in the included studies $(P<.01)$.

In an attempt to identify the source of heterogeneity in the summary estimate, we conducted sensitivity analyses by examining subgroups of studies with similar features, and by including some of the studies excluded from the main analysis (Table 2). In every combination that included more than 2 studies, heterogeneity was present. Despite the persistent heterogeneity, almost all of the subgroups showed a statistically significant benefit from NAC. In the analysis that included only randomized studies with a placebo control group there was a benefit from NAC, with a decrease in creatinine relative to placebo of -0.39 (95\% CI, -0.56 to -0.22$)$.

Nine randomized studies compared the incidence of CIN in patients treated with NAC versus controls. Combining these studies showed a summary relative risk (RR) of developing CIN of 0.51 (95\% CI, 0.35 to 0.74 ), indicating a statistically significant benefit from NAC (Fig. 2). Heterogeneity was also present in this summary measure $(P=.03)$. We again conducted sen- 
Table 1A. Study Characteristics

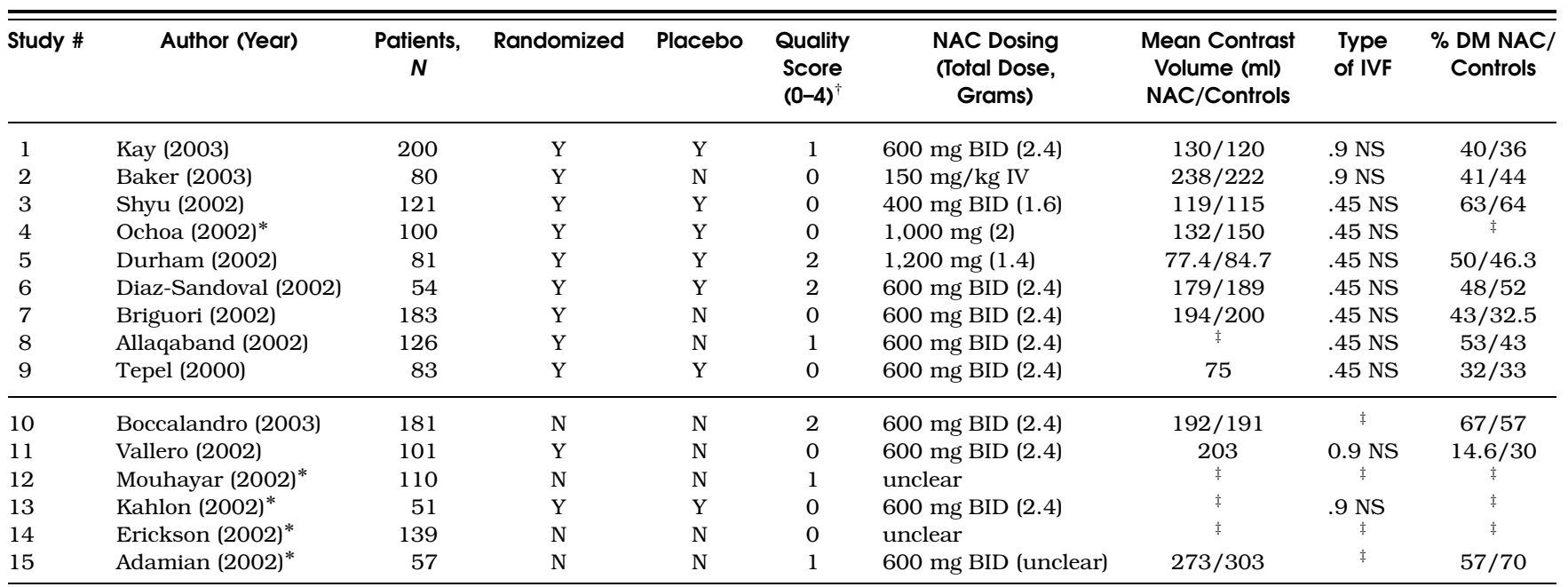

Studies included in the analysis are listed above the solid line, while excluded studies are listed below the solid line. All studies used IV hydration preand postcontrast administration. All studies enrolled $>90 \%$ of patients undergoing cardiac catheterization, except for Tepel et al., ${ }^{28}$ which enrolled patients undergoing CT scanning. Briguori et al. ${ }^{41}$ and Bader et al. ${ }^{40}$ are not included in the table because they both had another active treatment arm, but no control group. Kay et al. ${ }^{22}$ reported median contrast volume rather than mean contrast volume.

*Articles found only in abstract form.

Define quality score as 1 point for each of the following: appropriate randomization, allocation concealment, blinded patients, and blinded providers. ${ }^{\ddagger}$ Value unclear from provided data.

NAC, N-acetylcysteine; NS, normal saline; BID, twice daily; IVF, intravenous fluids; DM, diabetes.

Table 1B. Study Results

\begin{tabular}{|c|c|c|c|c|c|c|c|c|c|c|}
\hline Study \# & Author & $\begin{array}{c}\text { Mean Baseline } \\
\text { Cr, NAC } \\
\text { Group } \\
\text { (mg/dl) }\end{array}$ & $\begin{array}{c}\text { Mean Baseline } \\
\text { Cr, Control } \\
\text { Group } \\
\text { (mg/dl) }\end{array}$ & $\begin{array}{c}\text { Mean change } \\
\text { in } \mathrm{Cr} \text {, NAC } \\
\text { Group } \\
\text { (mg/dl) }\end{array}$ & $\begin{array}{c}\text { Mean change } \\
\text { in } \mathrm{Cr} \text {, Control } \\
\text { Group } \\
(\mathrm{mg} / \mathrm{dl})\end{array}$ & $\begin{array}{c}\text { Mean } \\
\text { Difference } \\
\text { Change in } \\
\text { Creatinine }^{\dagger}\end{array}$ & $\begin{array}{l}\% \text { CIN } \\
\text { NAC/ } \\
\text { Controls }\end{array}$ & $\begin{array}{l}\text { RR of } \\
\text { CIN }\end{array}$ & $\begin{array}{c}\text { Patients } \\
\text { Needing } \\
\text { Dialysis NAC/ } \\
\text { Controls, } N\end{array}$ & $\begin{array}{l}\text { Reason for } \\
\text { Exclusion }\end{array}$ \\
\hline 1 & Kay & 1.35 & 1.36 & -0.13 & +0.02 & -0.15 & $3.9 / 12.2$ & 0.32 & 0 & \\
\hline 2 & Baker & 1.85 & 1.75 & -0.08 & 0.05 & -0.14 & $4.9 / 20.4$ & 0.24 & 0 & \\
\hline 3 & Shyu & 2.80 & 2.80 & -0.29 & +0.24 & -0.53 & $3.3 / 24.6$ & 0.14 & $0 / 1$ & \\
\hline 4 & Ochoa* & 2.00 & 1.87 & 0.06 & +0.23 & -0.17 & $11.1 / 25.0$ & 0.44 & 0 & \\
\hline 7 & Briguori & 1.52 & 1.54 & -0.04 & -0.01 & -0.03 & $6.5 / 11.0$ & 0.59 & $0 / 1$ & \\
\hline 8 & Allaqaband & 2.20 & 2.03 & 0.01 & +0.09 & -0.08 & $17.8 / 15.0$ & 1.19 & $\ddagger$ & \\
\hline 9 & Tepel & 2.50 & 2.40 & -0.40 & +0.20 & -0.6 & $2.4 / 21.4$ & 0.11 & 0 & \\
\hline 10 & Boccalandro & 1.80 & 1.90 & 0.17 & +0.19 & -0.02 & $13.7 / 12.3$ & 1.12 & $\ddagger$ & Not randomized \\
\hline 11 & Vallero & $\ddagger$ & $\ddagger$ & $\ddagger$ & $\ddagger$ & N/A & $\ddagger$ & N/A & $\ddagger$ & $\begin{array}{l}\text { Groups separated } \\
\text { by Cr, varying } \\
\text { definitions of CIN }\end{array}$ \\
\hline 14 & Erickson* & $\ddagger$ & $\ddagger$ & $\ddagger$ & $\ddagger$ & N/A & $30 / 20$ & 1.69 & $6 / 1$ & Not randomized \\
\hline 15 & Adamian* & 1.89 & 1.96 & -0.01 & +0.52 & -0.53 & $2.9 / 36.4$ & 0.08 & $1 / 3$ & Not randomized \\
\hline
\end{tabular}

Studies included in the analysis are listed above the solid line, while excluded studies are listed below the solid line. All studies assessed time to outcome at 48 hours except Kahlon et al. ${ }^{30}$ which assessed outcome at 96 hours. Briguori et al. ${ }^{41}$ and Bader et al. ${ }^{40}$ are not included in the table because they both had another active treatment arm, but no control group.

*Articles found only in abstract form.

${ }^{\dagger}$ Negative values mean improvement in creatinine in NAC group compared to control group.

${ }^{\ddagger}$ Value unclear from provided data.

CIN, contrast-induced nephropathy; NAC, $\mathrm{N}$-acetylcysteine; $R R$, relative risk; $\mathrm{Cr}$, creatinine.

sitivity analyses to investigate sources of heterogeneity. As with the primary outcome measure, almost all subgroups showed a statistically significant benefit to NAC, and heterogeneity was present in each case when more than 2 studies were included (Table 3 ).
We found no evidence of publication bias when testing the primary outcome measure, the difference in mean change in creatinine between treatment groups $(P=.62$ for Begg's test, $P=.85$ for Egger's test; Fig. 3). When assessing for the presence of publication bias in the outcome of CIN, the results were 


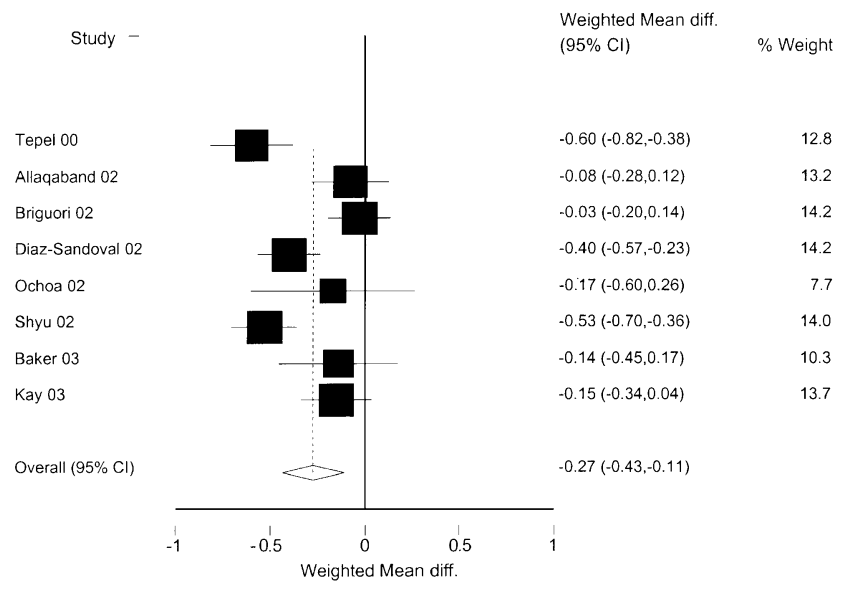

FIGURE 1. Weighted mean difference of change in creatinine between NAC and controls. Analysis based on random effects model. Units for weighted mean difference are $\mathrm{mg} / \mathrm{dl}$.

mixed. Analysis based on risk ratios showed evidence of publication bias $(P=.005$ for Begg's test, $P=.007$ for Egger's test), while analysis based on risk differences showed no publication bias $(P=.92$ for Begg's test, $P=.97$ for Egger's test). Funnel plots for CIN, which provide a visual format for examining the presence of publication bias, are shown in Figure 4.

Seven of the included studies reported information on dialysis, and 3 of the studies reported information on death. No patients required dialysis in the NAC group while 2 patients required dialysis in the no-NAC group, yielding an RR of 0.64 (95\% CI, 0.15 to 2.78$)$.

There were no deaths reported. Only 1 study reported information on length of hospitalization and oliguria. ${ }^{28}$ In this study, the mean length of hospitalization was 3.4 days in the NAC group and 3.9 days in the control group (RR, 0.52; $P=$.02). One patient in the NAC group and 3 patients in the control group developed oliguria (RR, 0.32; $P=0.29$ ).

Quality scores ranged from 0 to 2 out of 4 . The majority of studies did not provide sufficient information to establish whether they met at least some quality criteria.
In the subgroup of studies that included patients undergoing cardiac catheterization (all studies except Tepel et al. ${ }^{28}$ ), there was a benefit of NAC administration, with mean difference change in creatinine of $-0.22 \mathrm{mg} / \mathrm{dl}(95 \% \mathrm{CI},-0.38$ to -0.06 ) and an RR of developing CIN of 0.46 (95\% CI, 0.26 to 0.82). Significant heterogeneity was present for both outcome measures $(P<.01$ and $P=.04$, respectively).

\section{DISCUSSION}

Contrast-induced nephropathy is an important cause of acute renal failure and is associated with increased morbidity and mortality in hospitalized patients. ${ }^{15,42}$ Our analysis suggests that administration of NAC around the time of contrast administration prevents renal injury. Patients treated with NAC had both a lower mean creatinine (difference in mean $\Delta \mathrm{Cr}=-0.27 \mathrm{mg} / \mathrm{dl} ; 95 \% \mathrm{CI},-0.43$ to -0.11$)$ and a reduced risk of developing CIN compared with control patients (RR, 0.43 ; $95 \%$ CI, 0.24 to 0.75 ). In our sensitivity analyses, use of NAC was protective against renal injury in almost all subgroups that included at least 3 studies.

A particular strength of our study was our analysis of two separate outcome measures, both showing a benefit from NAC administration. Our use of a second outcome measure was particularly important given the varying definitions of CIN used in the studies. Some studies reported CIN as a rise in creatinine of $0.5 \mathrm{mg} / \mathrm{dl}$, while others reported CIN as a $25 \%$ increase in serum creatinine. Differing definitions of CIN make comparison between studies more difficult. Having change in creatinine as a second outcome measure allowed us to validate the findings of our first outcome measure, and provided an outcome measure that may be easier to interpret for most clinicians.

Despite the consistent results between the two outcome measures, the summary estimates must be interpreted with caution due to the presence of heterogeneity. We attempted to identify sources of heterogeneity by examining subgroups of studies with similar features. However, heterogeneity was present in almost all subgroups regardless of study characteristics including prevalence of diabetes, high baseline creatinine,

Table 2. Sensitivity Analysis for Primary Outcome, Mean Difference in Change in Creatinine

\begin{tabular}{|c|c|c|c|c|}
\hline Subgroups of Studies & Studies Included & Estimated Mean Difference in $\Delta \mathrm{Cr}(\mathrm{mg} / \mathrm{dl})$ & $95 \% \mathrm{Cl}$ & P Homogeneity* \\
\hline Studies included in meta-analysis & $1-4,6-9$ & -0.27 & -0.43 to -0.11 & $<.01$ \\
\hline High baseline $\mathrm{Cr}(\mathrm{Cr} \geq 2.0)$ & $3,4,8,9$ & -0.36 & -0.63 to -0.09 & $<.01$ \\
\hline Low baseline $\mathrm{Cr}(\mathrm{Cr}<2.0)$ & $1,2,6,7$ & -0.18 & -0.36 to 0.00 & .02 \\
\hline High mean contrast volume (vol $\geq 150 \mathrm{ml}$ ) & $2,6,7$ & -0.20 & -0.45 to 0.06 & .01 \\
\hline Low mean contrast volume (vol < $150 \mathrm{ml})$ & $1,3,4,9$ & -0.38 & -0.62 to -0.14 & $<.01$ \\
\hline High mean percentage with diabetes ( $\geq 50 \%$ ) & 3,6 & -0.46 & -0.59 to -0.33 & .29 \\
\hline Low mean percentage with diabetes $(<50 \%)$ & $1,2,7-9$ & -0.20 & -0.40 to 0.00 & $<.01$ \\
\hline Studies using 0.9 NS for hydration & 1,2 & -0.15 & -0.31 to 0.01 & .96 \\
\hline Studies using 0.45 NS for hydration & $3,4,6-9$ & -0.31 & -0.51 to -0.10 & $<.01$ \\
\hline All studies reporting mean difference ${ }^{\dagger}$ & $1-4,6-10,12,15$ & -0.28 & -0.43 to -0.13 & $<.01$ \\
\hline Randomized, placebo-controlled ${ }^{\dagger}$ & $1,3,4,6,9$ & -0.39 & -0.56 to -0.22 & .01 \\
\hline Studies using oral NAC formulation & $1,3-9$ & -0.29 & -0.46 to -0.11 & $<.01$ \\
\hline Studies using intra-arterial contrast & $1-8$ & -0.22 & -0.38 to -0.06 & $<.01$ \\
\hline
\end{tabular}

Mean difference change in creatinine, NAC versus placebo. Calculations were done using a random effects model. Study numbers are referenced from Table 1. The quality of studies was not included in the sensitivity analysis because only 1 study fit criteria for high quality. Studies using high doses of oral NAC were not analyzed because only 1 study fit criteria for high-dose oral NAC.

${ }^{*}$ Heterogeneity is present when $\mathrm{P}<.1$.

${ }^{\dagger}$ Analysis includes excluded studies.

Cr, creatinine; NAC, N-acetylcysteine; NS, normal saline; CI, confidence interval. 


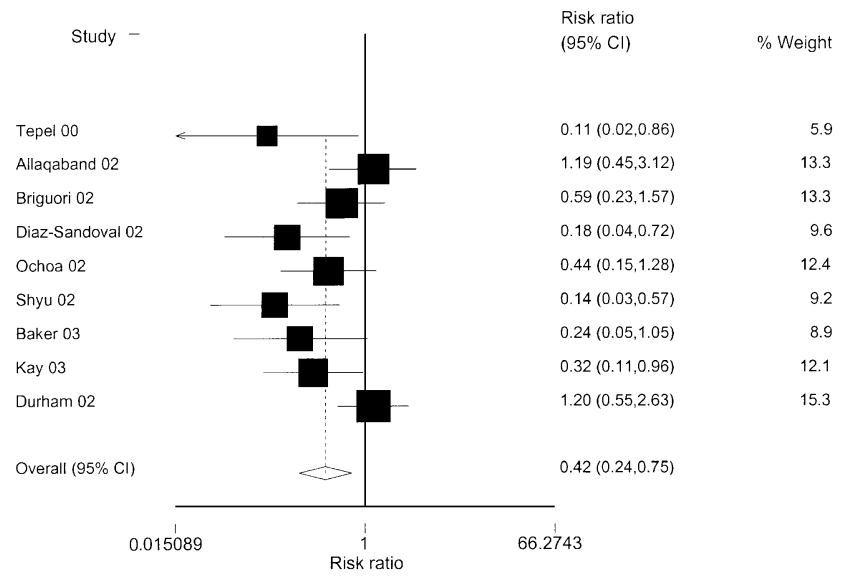

FIGURE 2. Relative risk of contrast-induced nephropathy between $\mathrm{N}$-acetylcysteine and control group. Analysis based on random effects model.

high- or low-contrast dose, and high and low study quality. In particular, sensitivity analysis eliminating the study using intravenous $\mathrm{NAC}^{29}$ or the study using intravenous rather than intra-arterial contrast ${ }^{28}$ failed to reduce the heterogeneity of the studies. The persistent heterogeneity suggests that there are differences in patient populations or study methodology that we were unable to identify. We were not able to perform sensitivity analysis on the different formulations of NAC as the specific oral formulation of NAC was not consistently reported in the studies.

Control of independent risk factors for renal dysfunction was not noted in most studies. For example, many of the medications that could affect renal function are not described clearly in the included studies. Medications such as furosemide may impair renal function in the setting of contrast administration. ${ }^{43}$ Other medications such as nonsteroidal anti-inflammatory drugs and angiotensin-converting enzyme inhibitor (ACEi) may affect renal function or the efficacy of NAC itself. Without more information, it is not possible to determine the contribution of these medications to the observed outcome measures.

Many of the studies did not receive high-quality scores. However, most of these studies did not specify whether or not they fit the quality criteria. The studies, therefore, could have had higher-quality scores than realized.

$\mathrm{N}$-acetylcysteine is inexpensive, easy to administer, and appears to have no significant toxicity. Our analysis suggests that it is effective for preventing the increase in serum creatinine caused by contrast administration, especially in the setting of cardiac catheterization. While the change in serum creatinine was only assessed at 48 hours, prior studies have documented that large changes in serum creatinine (CIN, or a change of $>0.5 \mathrm{mg} / \mathrm{dl}$ ) are associated with in-hospital morbidity and mortality. ${ }^{1,5-9}$ While the incidence is rare, it is also possible in those with advanced underlying renal disease that CIN could lead to a permanent loss of renal function and therefore earlier dialysis. ${ }^{44}$ Given the apparent low cost of NAC and the potential benefits, one might reasonably argue that NAC should be recommended for widespread use prior to contrast administration in all patients.

However, a major limitation of all of the included studies is the focus on the short-term changes in creatinine rather than on longer-term and more clinically relevant outcomes such as the incidence of dialysis and other adverse events during hospitalization, the length of hospitalization, and the rate of progression to end-stage renal disease after discharge. Only 1 study reported information on length of hospitalization and oliguria, and none of the studies reported the effect of NAC on overall costs or outcomes after discharge. Of the 7 studies that reported the incidence of dialysis among participants, dialysis was required in only 2 patients in the control group and in none in the NAC group. Although our meta-analysis had limited power to show a benefit from NAC administration in terms of preventing dialysis (due to the small number of patients requiring dialysis), our study did show that this outcome is rare $(0.2 \%)$. Therefore, when emergent intravenous contrast is indicated, our data suggest that there should

Table 3. Sensitivity Analysis for Secondary Outcome, CIN

\begin{tabular}{|c|c|c|c|c|}
\hline Subgroups of Studies & Studies Included & Summary RR & $95 \% \mathrm{Cl}$ & P Homogeneity* \\
\hline Studies included in meta-analysis & $1-9$ & 0.43 & 0.24 to 0.75 & .03 \\
\hline High baseline $\mathrm{Cr}(\mathrm{Cr} \geq 2.0)$ & $3-5,8,9$ & 0.50 & 0.21 to 1.20 & .02 \\
\hline Low baseline $\mathrm{Cr}(\mathrm{Cr}<2.0)$ & $1,2,6,7$ & 0.35 & 0.19 to 0.62 & .51 \\
\hline High quality $^{\dagger}$ & 5,6 & 0.51 & 0.08 to 3.24 & .02 \\
\hline Low quality & $1-4,7-9$ & 0.40 & 0.22 to 0.71 & .14 \\
\hline Studies using NAC $600 \mathrm{mg}$ BID $\times 4$ doses & $1,6-9$ & 0.42 & 0.20 to 0.90 & .09 \\
\hline Studies using high-dose PO NAC ( $\geq 1,000 \mathrm{mg} /$ dose $)$ & 4,5 & 0.78 & 0.30 to 2.04 & .14 \\
\hline High mean contrast volume (vol $\geq 150 \mathrm{ml}$ ) & $2,6,7$ & 0.35 & 0.16 to 0.74 & .32 \\
\hline Low mean contrast volume (vol $<150 \mathrm{ml})$ & $1,3-5,9$ & 0.37 & 0.16 to 0.88 & .03 \\
\hline High mean percentage with diabetes ( $\geq 50 \%$ ) & 3,6 & 0.16 & 0.06 to 0.42 & .79 \\
\hline Low mean percentage with diabetes $(<50 \%)$ & $1,2,5,7-9$ & 0.56 & 0.29 to 1.08 & .07 \\
\hline Studies using 0.9 NS for hydration & 1,2 & 0.29 & 0.12 to 0.70 & .75 \\
\hline Studies using $0.45 \mathrm{NS}$ for hydration & 3-9 & 0.46 & 0.23 to 0.91 & .02 \\
\hline All controlled studies reporting CIN & $1-10,14,15$ & 0.50 & 0.29 to 0.86 & $<.01$ \\
\hline Randomized, placebo-controlled & $1,3-6,9,13$ & 0.33 & 0.15 to 0.73 & .03 \\
\hline Studies using oral NAC formulation & $1,3-9$ & 0.45 & 0.24 to 0.82 & .03 \\
\hline Studies using intra-arterial contrast & $1-8$ & 0.46 & 0.26 to 0.82 & .04 \\
\hline
\end{tabular}

Relative risk of CIN, NAC versus placebo. Calculations were done using a random effects model. Study numbers are referenced from Table 1 .

${ }^{*}$ Heterogeneity is present when $\mathrm{P}<.1$.

Define quality as: explicitly complying with 2 or more of predefined quality features from abstraction form. These include appropriate randomization, allocation concealment, blinded patients, and blinded providers. Analysis includes excluded studies.

CIN, contrast-induced nephropathy; RR, relative risk; CI, confidence interval; Cr, creatinine; NAC, N-acetylcysteine; NS, normal saline; BID, twice daily; PO, by mouth. 


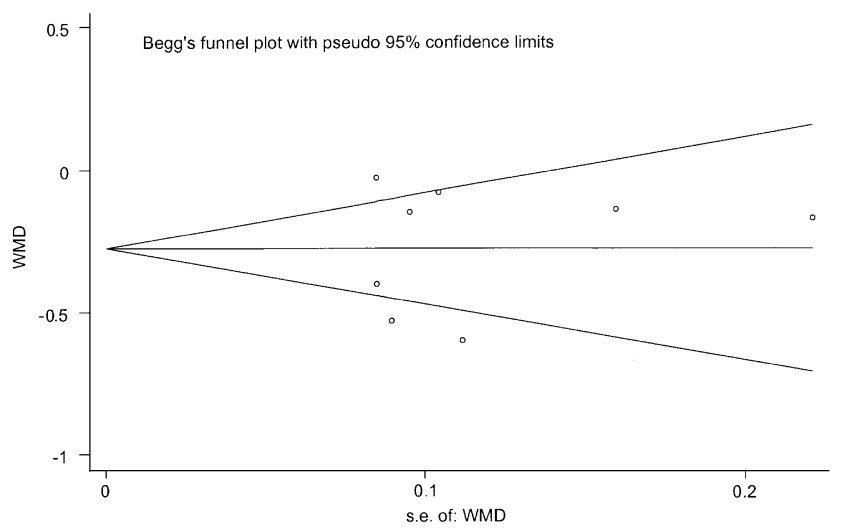

FIGURE 3. Funnel plot of weighted mean difference (WMD) versus standard error of WMD. There is no clear asymmetry around the summary estimate, indicating that the number of small negative and small positive studies is similar, and does not indicate the presence of publication bias for this outcome measure.

not be a delay in diagnostic or therapeutic procedures for administration of NAC. A related concern is that the widespread adoption of NAC may delay the identification and implementation of other potentially more beneficial interventions to reduce CIN.

To address the lack of information regarding clinically relevant outcomes, we believe that randomized controlled trials are needed to assess the effect of NAC on important long-term clinical outcomes and overall costs. Ideally, these studies should use a standardized dose and schedule of NAC, fluid hydration, contrast, and placebo, and should include outcomes such as renal failure, rates of dialysis, death, major cardiac events, and length of hospitalization. In addition, the baseline populations should be clearly defined in order to determine the effect of baseline renal function, diabetes, and concomitant medication use on outcomes.

A recently published systematic review concluded that NAC reduces the risk of CIN by $56 \%$ compared with control patients, but this study did not examine continuous outcomes (change in creatinine) or the incidence of dialysis among study patients. Furthermore, the results were limited by the finding of publication bias. In our study, we found no evidence of publication bias when examining our main outcome measure, the difference in mean change in creatinine between treatment groups. When assessing the presence of publication bias in the secondary outcome measure of CIN, we found publication bias only when using the same outcome measure as that used in the earlier study (i.e., relative risk; Fig. 4). This variability of results in tests of publication bias based on methodology has been described previously in the literature. ${ }^{45,46}$ While there is no evidence that one methodology of assessing publication bias is superior another, interpretation of publication bias based on risk differences would be in agreement with the results of our primary outcome measure. In our primary outcome measure, publication bias is not present and does not affect the accuracy of the summary estimate of the effect of NAC for the prevention of CIN.

Our study also identified 6 additional studies s,23,29-31,41 $^{2}$ not mentioned in the prior review. Two of these studies satisfied all inclusion criteria and were included in our metaanalysis. ${ }^{23,29}$ We also performed a more extensive sensitivity
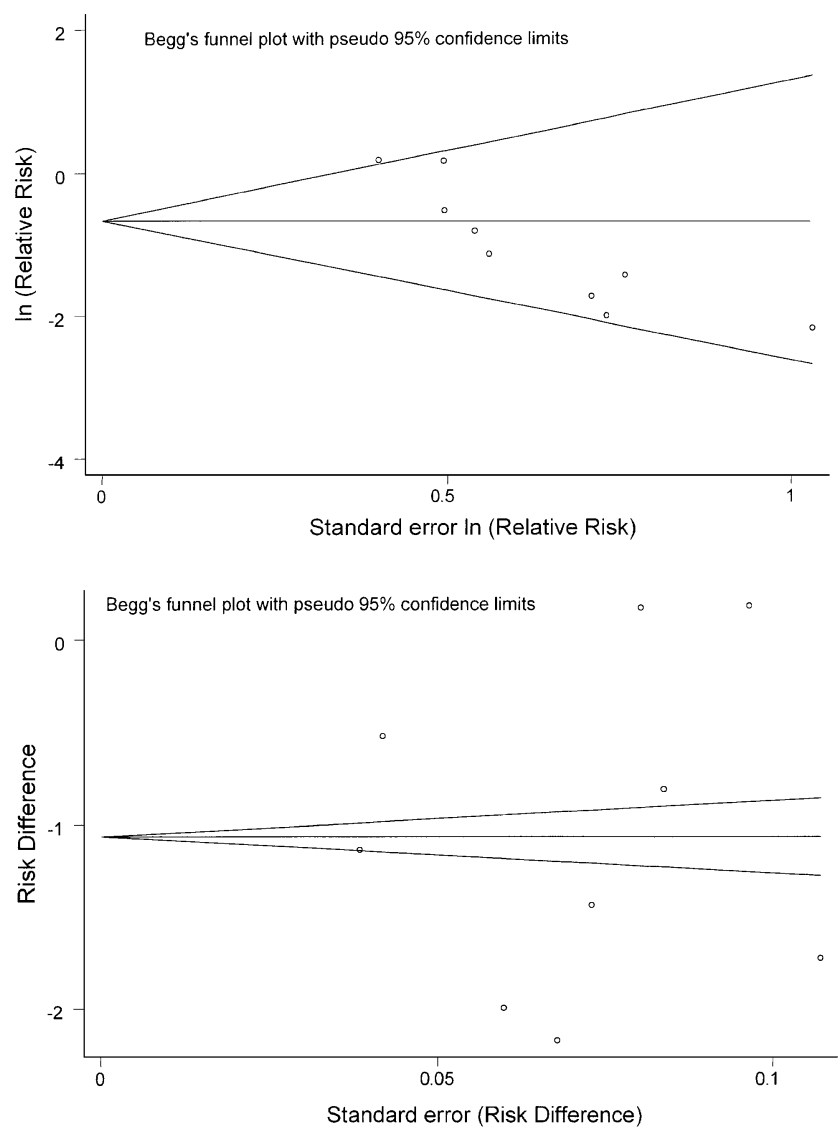

FIGURE 4. Funnel plots for outcome measure, contrast-induced nephropathy. The top funnel plot represents analysis based on risk ratios, showing asymmetry with a larger number of small positive studies (studies shown in lower right section of figure) compared to small negative studies (no studies in upper right section of figure), which suggests publication bias. The lower funnel plot represents analysis based on risk differences, showing lack of asymmetry and therefore suggesting lack of publication bias. The analysis based on risk differences is consistent with results shown in Figure 3.

analysis to examine the effect of patient characteristics and study design on heterogeneity, but were unable to identify the source of heterogeneity. However, the identification of 2 additional studies provided a more precise estimate of the effect of NAC in the subgroup of patients undergoing cardiac catheterization. We found a statistically significant benefit from NAC administration in these patients, while the prior study did not. ${ }^{47}$

There remain several important limitations to our metaanalysis, including the fact that many of the studies did not receive high-quality scores. Most of these studies did not specify whether or not they met the quality criteria, and the true quality of the studies therefore remains uncertain. Another important limitation is the inconsistent reporting among included studies of baseline patient characteristics and methods. In particular, lack of information regarding patient characteristics makes it difficult to identify subgroups that may or may not benefit from NAC. Furthermore, lack of more detailed information on the formulation of NAC used made it difficult to determine the optimal method of delivering the medication. Despite this limitation, we were able to use available data to identify a subgroup of patients 
undergoing cardiac catheterization that may benefit from NAC administration.

Finally, our ability to translate a reduced incidence of CIN and a lower mean creatinine to applicable clinical outcomes such as length of hospitalization and progression to end-stage renal disease is limited by the available data. In our analysis, the incidence of dialysis was rare, occurring in only $0.2 \%$ of patients. Further studies are needed to assess the impact of NAC on these useful clinical endpoints.

\section{Conclusions}

Our meta-analysis suggests that administration of NAC around the time of contrast delivery protects against worsening renal function and CIN at 48 hours. However, the incidence of dialysis is rare, and the long-term effect of this agent on more clinically important outcomes is not established. Widespread use of this agent has the potential to delay diagnostic or therapeutic interventions. Further studies are needed to assess the long-term effects of this agent and the overall costeffectiveness of routine NAC administration prior to contrast delivery, particularly in high-risk patients.

This work was supported by grant 1 K08 ATO1338-01 (Dr. Bent) from the National Center for Complementary and Alternative Medicine (NCCAM), and supported by funds from the PRIME Residency Program, University of California, San Francisco.

\section{REFERENCES}

1. McCullough PA, Wolyn R, Rocher LL, Levin RN, O'Neill Ww. Acute renal failure after coronary intervention: incidence, risk factors, and relationship to mortality. Am J Med. 1997;103:368-75.

2. Boccalandro F, Amhad $\mathbf{M}$, Smalling RW, Sdringola S. Oral acetylcysteine does not protect renal function from moderate to high doses of intravenous radiographic contrast. Catheter Cardiovasc Interv. 2003; 58:336-41

3. Parfrey PS, Griffiths SM, Barrett BJ, et al. Contrast material-induced renal failure in patients with diabetes mellitus, renal insufficiency, or both. A prospective controlled study. N Engl J Med. 1989;320:143-9.

4. Manske CL, Sprafka JM, Strony JT, Wang Y. Contrast nephropathy in azotemic diabetic patients undergoing coronary angiography. Am J Med. 1990;89:615-20.

5. Levy EM, Viscoli CM, Horwitz RI. The effect of acute renal failure on mortality. A cohort analysis. JAMA. 1996;275:1489-94.

6. Shusterman N, Strom BL, Murray TG, Morrison G, West SL, Maislin G. Risk factors and outcome of hospital-acquired acute renal failure. Clinical epidemiologic study. Am J Med. 1987;83:65-71.

7. Rihal CS, Textor SC, Grill DE, et al. Incidence and prognostic importance of acute renal failure after percutaneous coronary intervention. Circulation. 2002;105:2259-64.

8. Lindsay J, Apple S, Pinnow EE, et al. Percutaneous coronary intervention-associated nephropathy foreshadows increased risk of late adverse events in patients with normal baseline serum creatinine. Catheter Cardiovasc Interv. 2003;59:338-43.

9. Gruberg L, Mehran R, Dangas G, et al. Acute renal failure requiring dialysis after percutaneous coronary interventions. Catheter Cardiovasc Interv. 2001;52:409-16.

10. Davidson CJ, Hlatky M, Morris KG, et al. Cardiovascular and renal toxicity of a nonionic radiographic contrast agent after cardiac catheterization. A prospective trial. Ann Intern Med. 1989;110:119-24.

11. Rudnick MR, Goldfarb S, Wexler L, et al. Nephrotoxicity of ionic and nonionic contrast media in 1196 patients: a randomized trial. The Iohexol Cooperative Study. Kidney Int. 1995;47:254-61.

12. Baker CS, Baker LR. Prevention of contrast nephropathy after cardiac catheterisation. Heart. 2001;85:361-2.

13. Rudnick MR, Berns JS, Cohen RM, Goldfarb S. Nephrotoxic risks of renal angiography: contrast media-associated nephrotoxicity and atheroembolism-a critical review. Am J Kidney Dis. 1994;24:713-27.
14. Lautin EM, Freeman NJ, Schoenfeld AH, et al. Radiocontrast-associated renal dysfunction: incidence and risk factors. AJR Am J Roentgenol. 1991;157:49-58.

15. D'Elia JA, Gleason RE, Alday M, et al. Nephrotoxicity from angiograph ic contrast material. A prospective study. Am J Med. 1982;72:719-25.

16. Cigarroa RG, Lange RA, Williams RH, Hillis LD. Dosing of contrast material to prevent contrast nephropathy in patients with renal disease. Am J Med. 1989;86(pt 1):649-52.

17. Barrett BJ, Parfrey PS, Vavasour HM, et al. Contrast nephropathy in patients with impaired renal function: high versus low osmolar media. Kidney Int. 1992;41:1274-9.

18. Vlietstra RE, Nunn CM, Narvarte J, Browne KF. Contrast nephropathy after coronary angioplasty in chronic renal insufficiency. Am Heart J. 1996;132:1049-50.

19. Bakris GL, Lass N, Gaber AO, Jones JD, Burnett JC Jr. Radiocontrast medium-induced declines in renal function: a role for oxygen free radicals. Am J Physiol. 1990;258(pt 2):F115-F120.

20. Murphy ME, Tublin ME, Li S. Influence of contrast media on the response of rat renal arteries to endothelin and nitric oxide: influence of contrast media. Invest Radiol. 1998;33:356-65.

21. Zafarullah M, Li WQ, Sylvester J, Ahmad M. Molecular mechanisms of N-acetylcysteine actions. Cell Mol Life Sci. 2003;60:6-20.

22. Kay J, Chow WH, Chan TM, et al. Acetylcysteine for prevention of acute deterioration of renal function following elective coronary angiography and intervention: a randomized controlled trial. JAMA. 2003; 289:553-8.

23. Ochoa A, Isayenko Y, Pellizzon G, et al. Abbreviated dosing of N-acetylcysteine prevents contrast induced acute renal failure after coronary angiography and intervention. Abstract. Paper presented at American Heart Association meeting, Chicago, IL; November 5, 2002.

24. Adamian MG, Moussa I, Mehran R, et al. The role of mucormyst administration prior to percutaneous interventions on renal function in patients with chronic renal failure. Abstract. Paper presented at American College of Cardiology meeting, Atlanta, GA; March 6, 2002.

25. Mouhayar EN, Tadros G, Akinwande AAO, et al. Prevention of contrastinduced renal dysfunction with acetylcysteine in patients undergoing coronary angiography. Paper presented at American College of Cardiology meeting, Atlanta, GA; March 17, 2002.

26. Shyu KG, Cheng JJ, Kuan P. Acetylcysteine protects against acute renal damage in patients with abnormal renal function undergoing a coronary procedure. J Am Coll Cardiol. 2002;40:1383-8.

27. Diaz-Sandoval LJ, Kosowsky BD, Losordo DW. Acetylcysteine to prevent angiography-related renal tissue injury (the APART trial). Am J Cardiol. 2002;89:356-8.

28. Tepel M, van der Giet M, Schwarzfeld C, Laufer U, Liermann D, Zidek W. Prevention of radiographic-contrast-agent-induced reductions in renal function by acetylcysteine. N Engl J Med. 2000;343:180-4.

29. Baker CS, Wragg A, Kumar S, De Palma R, Baker LR, Knight CJ. A rapid protocol for the prevention of contrast-induced renal dysfunction: the RAPPID study. J Am Coll Cardiol. 2003;41:2114-8.

30. Kahlon J, Moser L, Rosman H, et al. Effectiveness of N-acetylcysteine for the prevention of radiocontrast-induced nephropathy: is the jury still out? Abstract. Paper presented at American Heart Association meeting, Chicago, IL; November 5, 2002.

31. Erickson C, Erickson J, Wilsker G, Brunner L. A retrospective analysis of oral acetylcysteine intervention to prevent radiographic contrast-induced nephropathy in patients undergoing coronary angiography with elevated serum creatinine. Abstract. Paper presented at NKF meeting, Chicago, IL; April 17, 2002.

32. Vallero A, Cesano G, Pozzato M, et al. [Contrast nephropathy in cardiac procedures: no advantages with prophylactic use of $\mathrm{N}$-acetylcysteine (NAC)]. G Ital Nefrol. 2002;19:529-33.

33. Durham JD, Caputo C, Dokko J, et al. A randomized controlled trial of $\mathrm{N}$-acetylcysteine to prevent contrast nephropathy in cardiac angiography. Kidney Int. 2002;62:2202-7.

34. Allaqaband S, Tumuluri R, Malik AM, et al. Prospective randomized study of $\mathrm{N}$-acetylcysteine, fenoldopam, and saline for prevention of radiocontrast-induced nephropathy. Catheter Cardiovasc Interv. 2002; 57:279-83.

35. Briguori C, Manganelli F, Scarpato P, et al. Acetylcysteine and contrast agent-associated nephrotoxicity. J Am Coll Cardiol. 2002;40: 298-303.

36. National Guideline Clearinghouse. Available at: www.guidelines.gov. Accessed August 15, 2003. 
37. Begg CB, Mazumdar M. Operating characteristics of a rank correlation test for publication bias. Biometrics. 1994;50:1088-101.

38. Egger M, Davey Smith G, Schneider M, Minder C. Bias in meta-analysis detected by a simple, graphical test. BMJ. 1997;315:629-34.

39. Higgins JP, Thompson SG. Quantifying heterogeneity in a meta-analysis. Stat Med. 2002;21:1539-58.

40. Bader B, Berger E, Rossmann S, Risler T, Erley C. Prevention of contrast media-induced acute renal failure in patients with severe impaired renal function-theophylline versus $\mathrm{N}$-acetylcysteine. Abstract. Paper presented at the Meeting of the American Society of Nephrology, Philadelphia, PA; November 2, 2002.

41. Briguori C, Colombo A, Violante A, et al. High versus low dose of acetylcysteine to prevent contrast agents associated with nephrotoxicity Abstract. Paper presented at Journal of the American College of Cardiology meeting, Chicago, IL; March 19, 2003.
42. Hou SH, Bushinsky DA, Wish JB, Cohen JJ, Harrington JT. Hospitalacquired renal insufficiency: a prospective study. Am J Med. 1983; 74:243-8.

43. Solomon R, Werner C, Mann D, D'Elia J, Silva P. Effects of saline, mannitol, and furosemide to prevent acute decreases in renal function induced by radiocontrast agents. N Engl J Med. 1994;331:1416-20.

44. Briguori C, Tavano D, Colombo A. Contrast agent-associated nephrotoxicity. Prog Cardiovasc Dis. 2003;45:493-503.

45. Tang JL, Liu JL. Misleading funnel plot for detection of bias in metaanalysis. J Clin Epidemiol. 2000;53:477-84.

46. Sterne JA, Egger M. Funnel plots for detecting bias in meta-analysis: guidelines on choice of axis. J Clin Epidemiol. 2001;54:1046-55.

47. Birck R, Krzossok S, Markowetz F, Schnulle P, van der Woude FJ, Braun C. Acetylcysteine for prevention of contrast nephropathy: metaanalysis. Lancet. 2003;362:598-603.

\section{SGIM $\mathrm{Q}$ th Annual Meeting \\ SGIM $28^{\text {th }}$ Annual Meeting \\ May 11-14, 2005 \\ New Orleans, Louisiana \\ Out of Chaos: The Critical Role of Generalists \\ Register Online at http://www.sgim.org/am}

VA-Related Programming:

Friday

1:00 pm-2:00 pm

SRF Workshop: Career Opportunities with the VA

Session Coordinator:

Jeffrey Whittle, MD, Kansas City VA Medical Center

Additional Faculty:

Gary Rosenthal, MD, lowa City VA Medical Center

Lisa Rubenstein, MD; VA Greater Los Angeles Health Care System

Ellen Yee, MD, Albuquerque VA Medical Center

Saturday

7:30-8:30 am

Interest Group IS10: VA PRIMARY CARE/SGIM INTEREST GROUP

Session Coordinator:

Lisa Rubenstein, MD, Professor of Medicine, VA Greater Los Angeles and UCLA

10:30AM - 12:00PM

Workshop G03: Improving Quality in Healthcare Systems: Does the VA Experience T ranslate to Other Healthcare Settings? Session Coordinator: Eve Kerr, MD, MPH, Associate Professor, VA Ann Arbor Health Care System and University of Michigan

These sessions are sponsored by an unrestricted educational grant from VA HSR\&D 